\title{
On single pole instrument voltage transformer overheating
}

\author{
František Janíček ${ }^{*}$, Lukáš Český ${ }^{1}$, Filip Skudřík ${ }^{2}$
}

\begin{abstract}
The paper deals with calculation of overheating of a voltage instrument transformer used in medium voltage substations. Calculation of the primary and secondary windings is compared with measured values. These values are evaluated according to IEC 618691:2007, IEC 61869-3:2011 and IEC 60085:2007. In evaluation we focused on the state $1.9 \times$ nominal voltage and thermal limiting output state. The article also compares the results for various parameters of instrument transformers that are necessary for clarification of calculation.

K e y w or ds: instrument transformer of voltage, overheating according to IEC 618693:2011, numerical model of overheating, IEC 60085:2007.
\end{abstract}

\section{Introduction}

Correct measuring of electrical parameters in a substation is necessary for a proper working of protection relays and billing of electric energy. Supplying of measuring and protecting devices with high values of voltages and currents is not optimal from the technical and economical point of view. This is the reason why it is necessary to transform these values to standardized values of voltage and current. For transformation, instrument transformers of voltage and current are used.

This paper is focused on single pole medium voltage instrument transformers of voltage. They are used mainly in medium voltage switchgears or laboratories. For a proper function of the voltage transformer it is necessary to take into consideration the operating parameters in the design and construction.

The article deals with calculation of overheating. Due to overheating of the active parts and insulation, damage or even destruction of the voltage transformer may occur. This is the reason why correct dimensions of active parts must be chosen. From the design point of view. for decreasing the overheating it is very important to choose sufficiently large cross-sections of the core, primary and secondary windings.

The main reason for needing a more accurate algorithm are even stricter requirements for overheating of switchgears. These changes are transmitted to manufacturers of instrument transformers. In standard IEC 60085:2007 temperature class $\mathrm{E}$ is defined. The maximal temperature of the object must not exceed $115{ }^{\circ} \mathrm{C}$. This value consists of ambient temperature and overheating by conditions described in IEC 61869:1-2007 and IEC 618693:2011. In these standards 3 states are described, when overheating is tested by $1.2 \times U_{n}$, by overvoltage factor usually $1.9 \times U_{\mathrm{n}} / 8 \mathrm{~h}$ and by thermal limiting output. For maximal values of overheating are important $1.9 \times U_{\mathrm{n}} / 8 \mathrm{~h}$ and thermal limiting output because the value of overheating by overvoltage factor is always higher than by $1.2 \times U_{\mathrm{n}}$. From the overheating point of view, the state with highest overheating is important [1-3].

According to standard IEC 61869-1:2007 in normal service condition the ambient temperature is $5^{\circ} \mathrm{C} / 40^{\circ} \mathrm{C}$. Connected with overheating temperature, this must not exceed temperature class E. Nowadays even higher requirements are imposed on ambient temperature in various applications, such as ambient temperature $80^{\circ} \mathrm{C}$ in generator circuit breakers. This means that the allowed value of overheating is reduced from $75^{\circ} \mathrm{C}$ to $35^{\circ} \mathrm{C}$, [1]

To obey the increased parameters it is necessary to refine overheating calculation. In comparison with power transformers the medium voltage transformer is usually casted in epoxy resin. With casting of epoxy resin shrinkage of the iron core is connected. This has a direct impact on the accuracy class. For elimination of core deformation, suspension and bandages are used. These are factors affecting thermal transmittance [4].

Our goal was the accuracy of $+15^{\circ} \mathrm{C}$ at the utmost in comparison with the measured values. The calculated value is always higher than the measured one. This is for safety reasons. The calculated values are compared with two measuring methods with thermal sensors and according to IEC standard. The paper also describes evaluation of overheating results studied in more samples with various configurations of parameters.

1 Slovak University of Technology in Bratislava, Faculty of Electrical Engineering and Information Technology, Ilkovičova 3, 81219 Bratislava, Slovakia, ${ }^{2}$ ABB, s.r.o., Vídeňská 117, 61900 Brno, Czech Republic, * Corresponding author: frantisek.janicek@stuba.sk 

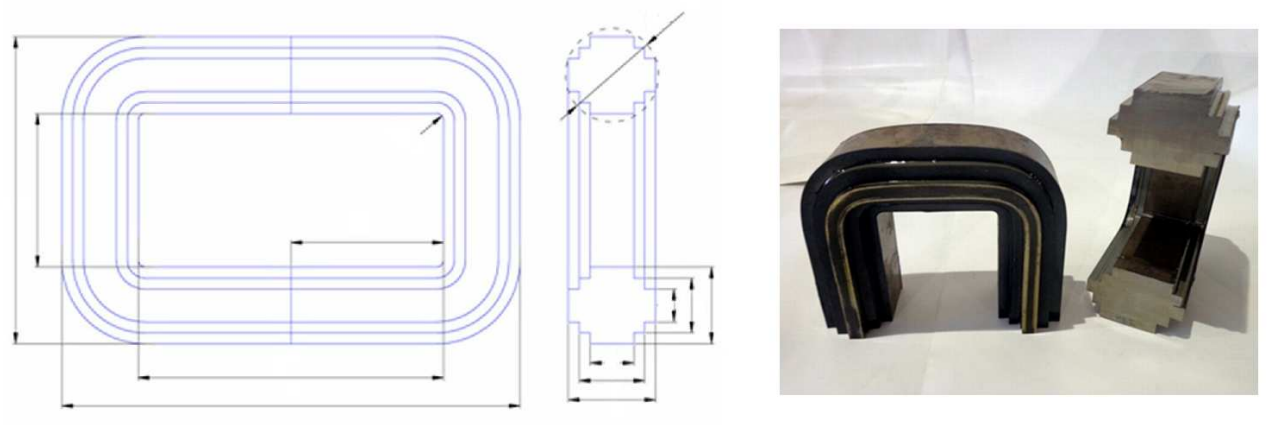

Fig. 1. Ferromagnetic core

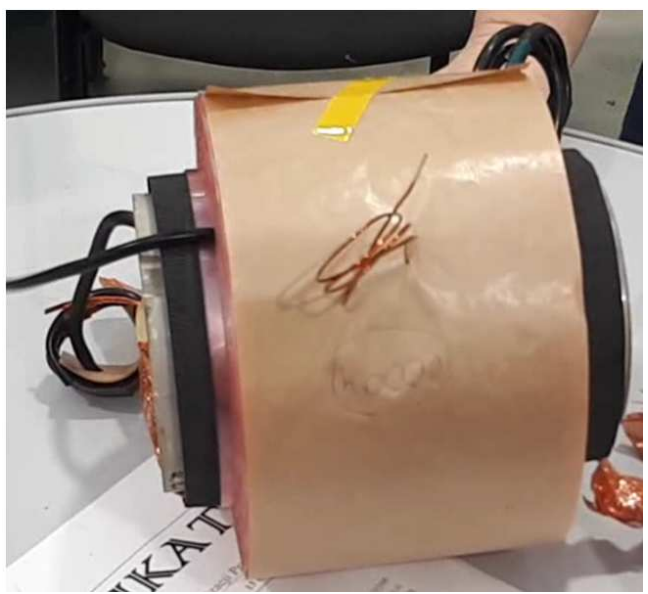

Fig. 2. Primary and secondary winding of experimental voltage transformer

\section{Design of active parts and overheating calculation}

Instrument transformer is a passive electrical device. For calculating its overheating it is necessary to know the design parameters including the ferromagnetic core and windings.

Maximal outer dimensions of instrument transformers are usually given by standards in virtue of unification for use in switchgears. In Europe, this is DIN standard [5].

In general, design of a transformer includes these issues: material of core and its dimensions depending on the maximal burden, number of primary turns according to rated magnetic induction $\mathrm{Bn}$, and of the secondary turns according to voltage ratio, dimension of wires for maximal current density $\left(\mathrm{eg} 3 \mathrm{~A} / \mathrm{mm}^{2}\right)$, and finally, the overheating estimation [6].

\subsection{The core}

In our case, the first task is the design of the ferromagnetic core of ferromagnetic steel with a thickness of 0.35 $\mathrm{mm}$ using a rectangular $\mathrm{C}$-core with three grades. Its cross section allows better filling of the secondary winding. The next benefits to be mentioned are [7] lower losses, reduction of used cooper due to smaller cores, easy assembly of coils on the core given by the cut core design, easier winding of the coil on the rectangular tube. The core used in this study is in Fig. 1.

Calculation of its cross section based on the Faraday law and reduced to the rated unity voltage is well known: $S_{\mathrm{FE}}=2 /\left(\omega B_{\mathrm{N}} N_{\mathrm{e}}\right)$, with: $\omega$ - rated angular frequency, $B_{\mathrm{n}}$ - rated magnetic induction, and secondary turns $N_{\mathrm{e}}$, This, not considering the attainable filling factor between $0.9<\eta<0.97$ (in our case 0.95 ) should be corrected to $S_{\mathrm{C}}=\eta S_{\mathrm{FE}}$.

From the overheating point of view, important are the so-called core surface cooling area $S \mathrm{C}$, and the environment conditions.

\subsection{The windings}

Windings of voltage instrument transformers in medium voltage networks usually consist of one primary and two secondaries. As a standard, secondary windings are placed on a secondary tube. First the measuring winding, and a protective winding onto it. On the secondary winding, separated by various types of interlayer insulation, the primary winding is coiled. Our experimental winding is in Fig. 2.

The numbers of primary $N_{1}$ turns and secondary $N_{2}$ are calculated by a standard method depending on the rated magnetic induction and the required output voltage.

The width $(D)$ of the coil depends on the diameter of the wire with insulation and the number of turns in one layer. The height of coil $(H)$ including the interlayer insulation gives the primary coil surface $S_{\mathrm{S}}=\pi D H$. The cooling surface of the secondary coil area is calculated analogously.

\subsection{Calculation of overheating}

The calculations were made concerning three different operation modes. First is continuous operation at $1.2 \times U_{\mathrm{n}}$. In this case the transformer works in the rated value conditions. The second mode is $1.9 \times U_{\mathrm{n}}$ for 8 hours. This is in a state of the network with an earth fault. The transformer must withstand rated voltage (factor $F_{\mathrm{v}}$ ). By earth fault works "open delta" winding, where the values of current are higher. The next reason overheating in state $1.9 \times U_{\mathrm{n}}$ is higher than in the continuous state is 
the overvoltage. When the instrument transformer works as a power supply, it is necessary to consider the highest possible burden. This burden is given mainly by the cross section of the core. According to standard IEC it is the thermal limiting output that is defined as a maximal burden that can be reached on the secondary winding of the transformer with accuracy $\pm 10 \%$. The thermal limiting output can only be connected to the measuring winding. In the protecting winding there must be 0 VA [4].

In the worst-case overheating of VT, the evaluation is done in: (i) thermal-limiting output state, and (ii) $1.9 \times U_{\mathrm{n}} / 8 \mathrm{~h}$. The values of all quantities in the continuous state are clearly the lowest. To examine the overheating calculation a voltage instrument transformer, type TJC 6, with rated voltage up to $24 \mathrm{kV}$ was used.

Parameters for overheating calculation are the dimensions of windings (thickness and width of coils, core cooling surface area, thermal limiting output in VA, primary and secondary resistance, and the thermal transmittance in $\left(\mathrm{W} / \mathrm{m}^{2} \mathrm{~K}\right)[4]$.

Overheating calculation is based on the Joule losses in windings and the magnetic core loss. The secondary current must be calculated for all modes of operation separately. The primary current is calculated from the rated voltages and secondary current. It is necessary to calculate it separately for all operation modes. If $I_{21}, I_{22}$ are the currents of the first/second secondary winding and $U_{21}, U_{22}$ are the voltages of the first secondary and the second secondary winding, for the primary voltage $U_{1}$, the primary current is

$$
I_{1}=I_{21} \frac{U_{21}}{U_{1}}+I_{22} \frac{U_{22}}{U_{1}} .
$$

In the next step we can calculate the Joule losses of the primary and secondary winding $\Delta P=R I^{2}, R$-being electrical resistance and $I$ the rms value of primary or secondary current, respectively.

The value of the core losses per $\mathrm{kg}$ is provided by the manufacturer as a function of induction. In calculation of core losses, we have considered the values appropriate to a particular mode of operation. Thus as uncorrected value of overheating can be taken $\Delta \nu=\Delta P /(\alpha S)$, where $\alpha$ is the thermal transmittance over the cooling surface $S$.

These values should be correct in an ideal case without effects between all active parts of the voltage instrument transformer and with thermal transmittance calculated as constant for the epoxy resin. However, in a real instrument transformer of voltage the influence should be considered of various materials used in active parts, such as cooper wire, interlayer insulation or epoxy resin. This is the reason why thermal transmittance for each winding was determined separately. After correction of $\alpha$ coefficient the effect of overheating of the active parts was calculated for each winding and for the ferromagnetic core $\Delta \nu_{\text {cor }}\left(\mathrm{W} / \mathrm{m}^{2} \mathrm{~K}\right)[3]$.

\section{Comparison of calculation and measurement}

Calculated overheating was compared with the measurement made by two different methods. Resistance method described in IEC standard, and measuring with thermal sensors. The values according to IEC resistance method were considered as reference values. Calculated values are in Tab. 1.

Table 1. The overheating calculation $\Delta \nu$ in Kelvins

\begin{tabular}{|c|c|c|}
\hline \multirow{2}{*}{$\begin{array}{l}\text { Overheating } \\
\text { of windings }\end{array}$} & \multicolumn{2}{|c|}{ Operation mode } \\
\hline & $500 \mathrm{VA}$ & $1.9 \times U_{n}$ \\
\hline primary & 23.86 & 20.15 \\
\hline 1st secondary & 28.17 & 23.6 \\
\hline 2nd secondary & 27.77 & 23.0 \\
\hline
\end{tabular}

The temperature rise test is described in standard IEC 61869-3, clause 7.2.2. In the standard there are described three operation states. Test of states $1.2 \times U_{\mathrm{n}}$ and thermal limiting output by $1 \times U_{\mathrm{n}}$ is till stability of overheating. Test by $\mathrm{Fv}$, in our case $1.9 \times U_{\mathrm{n}}$ in duration of $8 \mathrm{~h}$. Thermal limiting output is connected always to the measuring winding, not the protective one. If a transformer has more than one measuring winding, each winding should withstand separately the thermal limiting output. In this state the other windings remain unloaded.

In our experiments the ambient temperature range was $-5^{\circ} \mathrm{C}$ to $40^{\circ} \mathrm{C}$ and insulation class E. This means maximal overheating $50^{\circ} \mathrm{C}$. We have used the following equipment: high voltage test system WGBS, HIGHVOLT, ohm-meter GOM 802, measuring of temperature and humidity Tecpel RS 322, measuring of temperature, humidity and pressure HTPM 27/350, ampere meters FL 11, multimeter TECPEL DMM 8050.

Testing was provided by measuring the resistance for each winding before the test and at the end of test. Ambient air conditions during the tests were as follows: ambient temperature before test $22.4^{\circ} \mathrm{C}$, ambient temperature after test $24.4^{\circ} \mathrm{C}$, air pressure $999 \mathrm{hPa}$ and relative humidity $25 \%$ for $1.9 \times U_{\mathrm{n}} / 8 \mathrm{~h}$ state and ambient temperature before test $22^{\circ} \mathrm{C}$, ambient temperature at the end of test $24.1^{\circ} \mathrm{C}$, air pressure $978 \mathrm{hPa}$ and relative humidity for thermal limiting output test. Overheating is given as

$$
\Theta=\frac{R-R_{0}}{R_{0}}\left(235-\nu_{0}\right)-\left(\nu-\nu_{0}\right)
$$

where, $R_{0}$ and $\nu_{0}$ are the winding resistance and the ambient temperature before the test, while $\nu$ and $R$ are the values after the test.

The first test was performed for voltage $1.2 \times U_{n}$, immediately after this a test was conducted by voltage factor $F_{v} 1.9 \times U_{n}$ for 8 hours under the $24.1 \mathrm{kV}$ and rated burden on both secondary windings $-50 / 50 \mathrm{VA}$. The 
Table 2. Temperature rise by $1.9 \times U_{\mathrm{n}}$

\begin{tabular}{lccc}
\hline Winding & $\begin{array}{c}R_{0} \\
(\Omega)\end{array}$ & $\begin{array}{c}R \\
(\Omega)\end{array}$ & $\begin{array}{c}\text { Temperature } \\
\text { rise }(\mathrm{K})\end{array}$ \\
\hline $\mathrm{A}-\mathrm{N}$ & 2843 & 3052 & 16.9 \\
$\mathrm{a}-\mathrm{n}$ & 0.129 & 0.142 & 23.4 \\
$\mathrm{da}-\mathrm{dn}$ & 0.137 & 0.150 & 22.7 \\
\hline
\end{tabular}

Table 3. Temperature rise by $1.0 \times U_{\mathrm{n}}$

\begin{tabular}{lccc}
\hline Winding & $\begin{array}{c}R_{0} \\
(\Omega)\end{array}$ & $\begin{array}{c}R \\
(\Omega)\end{array}$ & $\begin{array}{c}\text { Temperature } \\
\text { rise }(\mathrm{K})\end{array}$ \\
\hline $\mathrm{A}-\mathrm{N}$ & 2833 & 3056.0 & 18.6 \\
$\mathrm{a}-\mathrm{n}$ & 0.1299 & 0.1420 & 22.3 \\
$\mathrm{da}-\mathrm{dn}$ & 0.1372 & 0.1500 & 22.3 \\
\hline
\end{tabular}

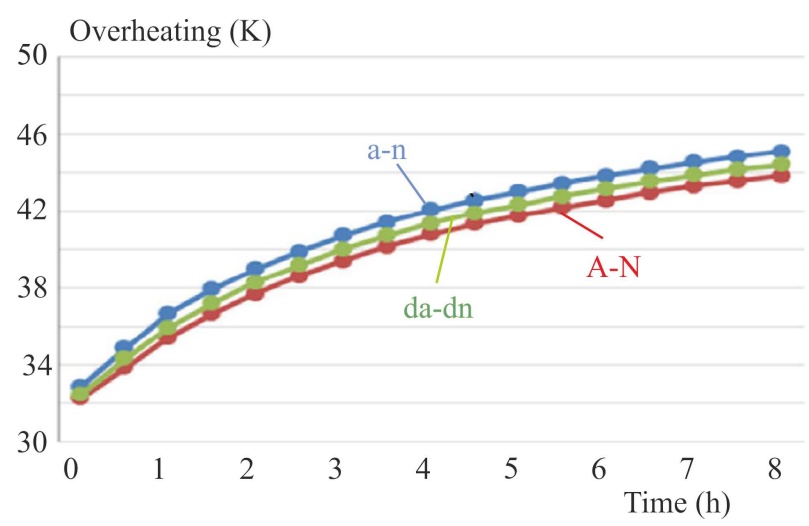

Fig. 3. Overheating in time by $1.9 \times U_{\mathrm{n}}$

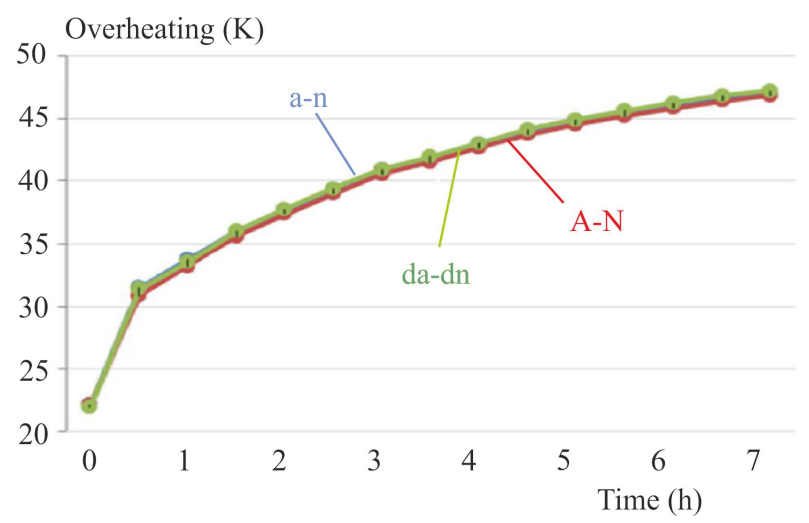

Fig. 4. Overheating in time by $1.0 \times U_{\mathrm{n}}$

resistance before $R_{0}$, and at the end of the test $R$ and temperature rise are in Tab. 2.

Temperature rise test by thermal limiting output was tested by $1.0 \times U_{\mathrm{n}}$. With a burden of $500 \mathrm{VA}$ on the first secondary and $0 \mathrm{VA}$ on the second secondary. Resistances before and after the test and temperature rise are given in Tab. 3.

Voltage transformer passed temperature rise test for all operation states in insulation class $\mathrm{E}$ with ambient temperature $-5^{\circ} \mathrm{C}+40^{\circ} \mathrm{C}$ according to IEC $61869-3$, clause 7.2.2.
For verification of results thermal sensors HEL-707T-0-12-00 were casted in the voltage transformer. The measuring range is between $-75^{\circ} \mathrm{C}$ and $+540^{\circ} \mathrm{C}$, the temperature coefficient is $0.00385 \Omega /{ }^{\circ} \mathrm{C}$. The difference between the resistance method and using of thermal sensors is in the measuring logic. The resistance method considers the average value of temperature rise in the whole winding. Thermal sensors measure the spots, where they are placed, in our case between the primary and secondary winding, in the start of the first and second secondary windings. This is the reason why deviations may occur in results between these two methods. Deviation should appear mainly on the primary winding where in our case there are 72 layers of wire and interlayer insulation. Thermal sensors were connected to the secondary terminal [3].

By testing of $1.9 \times U_{\mathrm{n}}$ the temperature rise of the primary winding $\mathrm{A}-\mathrm{N}$ was $22.4 \mathrm{~K}$, temperature rise of the first secondary winding $\mathrm{a}-\mathrm{n} 23.6 \mathrm{~K}$ and temperature rise of the second secondary winding $23.1 \mathrm{~K}$. Overheating versus time is in Fig. 3 .

By testing of $1.0 \times U_{\mathrm{n}}$ the temperature rise of primary winding $\mathrm{A}-\mathrm{N}$ was $24.7 \mathrm{~K}$, temperature rise of the first secondary winding a-n $25.0 \mathrm{~K}$ and temperature rise of the second secondary winding $25.3 \mathrm{~K}$. Overheating versus time is in Fig. 4.

Here we compare the results of overheating obtained by calculation and measurement. The resistance method is recommended by standards as a verification tool. The value of overheating can by checked by the manufacturer and customer by the same routings.

Calculation was considered as a reference value. Our goal was the difference between calculation and the resistance method to be $15 \mathrm{~K}$ at the utmost. The calculated value should be always higher than measured one because of the safety margin.

Table 4. Results of overheating by $1.9 \times U_{\mathrm{n}}$ for $8 \mathrm{~h}$

\begin{tabular}{lccc} 
Winding & Calculation & Resistance & Sensor \\
\hline A-N & 20.15 & 16.9 & 22.4 \\
a-n & 23.6 & 23.4 & 23.6 \\
da-dn & 23.0 & 22.7 & 23.1 \\
\hline
\end{tabular}

Table 5. Difference between methods by $1.9 \times U_{n}$ for $8 \mathrm{~h}$

\begin{tabular}{lcc} 
Winding & Resistance & Sensor \\
\hline $\mathrm{A}-\mathrm{N}$ & -3.25 & 2.25 \\
$\mathrm{a}-\mathrm{n}$ & -0.19 & 0.002 \\
$\mathrm{da}-\mathrm{dn}$ & -0.305 & 0.095 \\
\hline
\end{tabular}

The difference between calculation and resistance method was maximal $3.2 \mathrm{~K}$. Calculation reached the goal of maximally $15 \mathrm{~K}$ and higher value by calculation than by measuring. The values of overheating measured with thermal sensors are higher than calculation. As written 
Table 6. Results of overheating by $1.0 \times U_{\mathrm{n}}$

\begin{tabular}{lccc} 
Winding & Calculation & Resistance & Sensor \\
\hline A-N & 23.86 & 18.6 & 24.7 \\
a-n & 28.17 & 22.3 & 25.0 \\
da-dn & 27.8 & 22.3 & 25.3 \\
\hline
\end{tabular}

Table 7. Difference between methods by $1.0 \times U_{\mathrm{n}}$

\begin{tabular}{lcc} 
Winding & Resistance & Sensor \\
\hline $\mathrm{A}-\mathrm{N}$ & -5.26 & 0.84 \\
$\mathrm{a}-\mathrm{n}$ & -5.87 & -3.17 \\
$\mathrm{da}-\mathrm{dn}$ & -5.47 & -2.47 \\
\hline
\end{tabular}

Table 8. Overheating of TJP $6.0-\mathrm{G}, 1.9 \times U_{n}$

\begin{tabular}{lccc}
\hline Winding & Calculation & Resistance & Difference \\
A-N & 21.72 & 15.5 & 6.22 \\
$\mathrm{a}-\mathrm{n}$ & 22.72 & 12.9 & 9.82 \\
da-dn & 22.76 & 18.7 & 4.06 \\
\hline
\end{tabular}

Table 9. Overheating of TJP $6.0-\mathrm{G}, 1.0 \times U_{n}$

\begin{tabular}{lccc}
\hline Winding & Calculation & Resistance & Difference \\
A-N & 28.98 & 21.2 & 7.78 \\
$\mathrm{a}-\mathrm{n}$ & 26.26 & 17.4 & 8.9 \\
da-dn & 26.67 & 16.7 & 9.9 \\
\hline
\end{tabular}

before, this is caused by measuring of one spot versus average overheating. Results of overheating for all three methods by $1.0 \times U_{n}$ by thermal limiting output are in Tab. 6. Table 7 shows the differences between the measuring methods and calculation.

Difference between calculation and resistance method for this case was maximal $5.2 \mathrm{~K}$. Calculation reached the goal of maximally $15 \mathrm{~K}$ and a higher value by calculation than by measuring.

\section{Evaluation of overheating calculation}

Three voltage instrument transformers were tested with different parameters as the ratio, rated burden or another transformer type. This implies another configuration of active parts and the volume of the epoxy resin. In the next step the voltage transformer from chapter three was tested repeatedly but with another rated burden 100/150 VA. Overheating was first calculated and afterwards measured according to IEC 61869-3, clause 7.2.2.

The first tested instrument was ABB transformer TJP 6.0-G with parameters: $500 \mathrm{VA}, 22000 / \sqrt{3} / / 110 / \sqrt{3} /$ $110 / 3 \mathrm{~V}, 50 \mathrm{~Hz},-5+40^{\circ} \mathrm{C}, 24 / 50 / 125 \mathrm{kV}$. Values for state $1.0 \times U_{n}$ are in Tab. 9. Calculation provided higher values than measurements in all cases and the maximal difference between the methods was $9.9 \mathrm{~K}$.

The next tested was transformer TJP 4.4 with parameters: $11000 / \sqrt{3} / / 110 / \sqrt{3} / 110 / 3 \mathrm{~V}, 50 \mathrm{~Hz},-5+65^{\circ} \mathrm{C}$, $12 / 42 / 75 \mathrm{kV}$. Calculation values were higher than measured ones in all cases and the maximal difference between the methods was $10 \mathrm{~K}$.

Table 10. Overheating of TJP 4.4, $1.9 \times U_{\mathrm{n}}$ for $8 \mathrm{~h}$

\begin{tabular}{lccc}
\hline Winding & Calculation & Resistance & Difference \\
$\mathrm{A}-\mathrm{N}$ & 22.80 & 18.1 & 4.70 \\
$\mathrm{a}-\mathrm{n}$ & 23.71 & 13.7 & 10.00 \\
$\mathrm{da}-\mathrm{dn}$ & 23.24 & 23.1 & 0.14 \\
\hline
\end{tabular}

The values for state $1.0 \times U_{\mathrm{n}}$ are in Tab. 11 .

Table 11. Overheating of TJP 4.4, $1.0 \times U_{\mathrm{n}}$

\begin{tabular}{lccc}
\hline Winding & Calculation & Resistance & Difference \\
A-N & 24.03 & 17.9 & 6.13 \\
a-n & 26.63 & 16.9 & 9.73 \\
da-dn & 26.76 & 22.8 & 3.96 \\
\hline
\end{tabular}

The last tested was TJC $6-\mathrm{G}$ with parameters: $20000 / \sqrt{3} / / 110 / \sqrt{3} / 110 / 3 \mathrm{~V}, \quad 50 \mathrm{~Hz}, \quad-5+40{ }^{\circ} \mathrm{C}$, $24 / 60 / 125 \mathrm{kV}$.

Values for state $1.0 \times U_{\mathrm{n}}$ are in Tab. 13. Calculation values were higher than the measured ones in all cases and the maximal difference between the methods was $13.4 \mathrm{~K}$. Values for state $1.0 \times U_{\mathrm{n}}$ are in Tab. 15. Calculation values were higher than measured in all cases and the maximal difference between the methods was $7.7 \mathrm{~K}$. All calculated values in evaluation passed required conditions.

\section{Conclusion}

Our goal was evaluation of an algorithm for overheating calculation of a single pole medium voltage instrument transformer. Our reference value for comparison with calculated values was the measuring method of winding resistance according to IEC 61869-3, clause 7.2.2. From maximal overheating point of view two operation states were important: $1.9 \times U_{\mathrm{n}} / 8$ hours (voltage factor) and $1.0 \times U_{\mathrm{n}}$ by thermal limiting output connected on the measuring winding.

For verifying the results we casted thermal sensors Honeywell Hell series into one voltage transformer. It was placed at the start of each winding. Sensors were connected to the secondary terminal. In comparison with the resistance method, the sensor calculates with temperature rise in one spot, whereas the resistance method 
Table 12. Overheating of TJC 6-G, $1.9 \times U_{\mathrm{n}}$

\begin{tabular}{lccc}
\hline Winding & Calculation & Resistance & Difference \\
A-N & 28.73 & 15.3 & 13.43 \\
a-n & 26.93 & 18.8 & 8.13 \\
da-dn & 26.91 & 19.3 & 7.61 \\
\hline
\end{tabular}

Table 13. Overheating of TJC 6-G, $1.0 \times U_{\mathrm{n}}$

\begin{tabular}{lccc}
\hline Winding & Calculation & Resistance & Difference \\
$\mathrm{A}-\mathrm{N}$ & 26.83 & 22.1 & $4.73 \mathrm{~K}$ \\
$\mathrm{a}-\mathrm{n}$ & 28.66 & 23.4 & $5.26 \mathrm{~K}$ \\
$\mathrm{da}-\mathrm{dn}$ & 28.76 & 23.8 & $4.96 \mathrm{~K}$ \\
\hline
\end{tabular}

Table 14. Overheating of TJC $6-\mathrm{G}, 1.9 \times U_{\mathrm{n}}$ for $8 \mathrm{~h}$

\begin{tabular}{lccc}
\hline Winding & Calculation & Resistance & Difference \\
$\mathrm{A}-\mathrm{N}$ & 45.52 & 37.82 & 7.70 \\
$\mathrm{a}-\mathrm{n}$ & 41.89 & 37.59 & 4.30 \\
$\mathrm{da}-\mathrm{dn}$ & 43.25 & 39.34 & 3.91 \\
\hline
\end{tabular}

Table 15. Overheating of TJC 6-, $1.0 \times U_{\mathrm{n}}$

\begin{tabular}{lccc}
\hline Winding & Calculation & Resistance & Difference \\
$\mathrm{A}-\mathrm{N}$ & 23.857 & 22.71 & 1.147 \\
$\mathrm{a}-\mathrm{n}$ & 28.166 & 22.98 & 5.186 \\
$\mathrm{da}-\mathrm{dn}$ & 27.770 & 23.12 & 4.65 \\
\hline
\end{tabular}

according to standard deals with an average value of temperature rise. This is the reason of deviations between these two methods. Deviation is obvious mainly in the primary winding. This is due to the geometry of windings - primary winding has usually 70 or more layers of wire and interlayer insulation. The secondary winding has only 2 to 5 layers.

Our main goal was calculating the temperature rise with deviation of $+15 \mathrm{~K}$ at the utmost. Calculated value should be always higher than the measured due to safety margin. We reached our goal in all cases.

Calculation of overheating is based on the Joule losses on the windings and core losses on the ferromagnetic core. Losses together with the cooling surface area and thermal transmittance give an uncorrected value of overheating. For a real value it is necessary to correct the value of thermal transmittance given by various materials used in the voltage transformer such as epoxy resin, interlayer insulation, cooper wire, semi conductive layer, etc. It is also necessary to consider the influence between active parts (between primary and secondary windings or between windings and core).

At the end of the article three instrument transformers of voltage were calculated and measured and the transformer from chapter two remeasured for rated burden $100 / 150$ VA. The main purpose was evaluation of overheating calculation in various types of voltage transform- ers. Each transformer had a different configuration of active parts and different mass of the epoxy resin.

\section{Acknowledgements}

This work was supported by the Operational Program Integrated Infrastructure for the project International Center of Excellence for Research on Intelligent and Secure Information and Communication Technologies and Systems - 2nd stage, ITMS code: 313021W404, cofinanced by the European Regional Development Fund.

\section{REFERENCES}

[1] IEC 61869-1:2007 Instrument Transformers - part 3: General Requirements, 1 st edition, Geneva, 2007.

[2] IEC 61869-3:2011 Instrument Transformers - part 3: Adittional Requirements for Inductive Voltage Transformers, 1 st edition, Geneva, 2011, 28 p.

[3] IEC 60085:2007 Electrical Insulation - Thermal Evaluation and Designation, 4 th edition, Geneva, 2007.

[4] L. Cesky, F. Janicek, J. Kubica, and F. Skudrik, "Overheating of Primary and Secondary Coils of Voltage Instrument Transformers", 18 th International Scientific Conference on Electric Power Engineering (EPE), Kouty nad Desnou, 2017, pp. 1-6, ISBN: 978-1-5090-6405-2.

[5] DIN 42600-8:2013-10 Messwandler für $50 \mathrm{~Hz}, U_{\mathrm{m}} 0,72 \mathrm{kV}$ bis $52 \mathrm{kV}$ - Teil 8: Stützer-Stromwandler $12 \mathrm{kV}$ und $U_{\mathrm{m}}=24 \mathrm{kV}-$ Schmale Bauform, Hauptmasse, Innenraumausführung, Berlin, 2013, 5p.

[6] M. Patocka, Magnetické jevy a obvody ve výkonové elektronice, měřicí technice a silnoproudé elektrotechnice, University of Technology Brno, 2011, (in Czech).

[7] P. Velesik and J Vanek, Instrument voltage transformers of outdoor type, Master thesis, University of Technology Brno, 2008.

[8] Dělená C jádra, Liberty Ostrava, https://libertyostrava.cz/ospolecnosti/zavody/liberty-technotron-s-r-o/produkty/delena -c-jadra [03.11.2020].

Received 29 October 2020

František Janíček (prof, Ing, PhD) was born in 1954. He is director of the Institute of Power and Applied Electrical Engineering at the Faculty of Electrical Engineering and Information Technology (FEI) of the Slovak University of Technology (SUT) in Bratislava. His research is focused on electrical protections, power stations, substations, energy problems and renewable energy sources.

Lukáš Český (Ing), born in 1992, graduated from the Faculty of Electrical Engineering and Information Technology of the Slovak University of Technology in 2016. Currently he works as a project engineer in ABB Brno s.r.o. He is responsible for $\mathrm{R} \& \mathrm{D}$ of medium voltage instrument transformers. His research activities are focused on overheating of MV instrument transformers of voltage.

Filip Skudřík (Ing), born in 1987, graduated from the Faculty of Electrical Engineering and Computer Science at VSB Technical university of Ostrava in 2011. At this moment he works as Engineering and Technology manager for Instrument transformers and sensors in ABB s.r.o. Brno. He is responsible for order engineering, $R \& D$ engineering and mould engineering for instrument transformers and sensors. 